\title{
A necessary and sufficient condition for the strict stationarity of a family of GARCH processes*
}

\author{
Mika Meitz \\ Stockholm School of Economics
}

SSE/EFI Working Paper Series in Economics and Finance No. 601

July 23, 2005

\begin{abstract}
We consider a family of $\operatorname{GARCH}(1,1)$ processes introduced in He and Teräsvirta (1999a). This family contains various popular GARCH models as special cases. A necessary and sufficient condition for the existence of a strictly stationary solution is given.
\end{abstract}

\section{Introduction}

He and Teräsvirta (1999a) considered a general class of first-order GARCH models and examined the moment structure within this family. In their paper, the sequence of random variables $\left\{\varepsilon_{t}\right\}_{t=-\infty}^{\infty}$ belongs to this general class of $\operatorname{GARCH}(1,1)$ processes if

$$
\begin{aligned}
\varepsilon_{t} & =z_{t} h_{t} \\
h_{t}^{k} & =g\left(z_{t-1}\right)+c\left(z_{t-1}\right) h_{t-1}^{k}
\end{aligned}
$$

where $\left\{z_{t}\right\}$ is a sequence of independent and identically distributed random variables with zero mean, $k$ equals 1 or 2 , and $g_{t}=g\left(z_{t}\right)$ and $c_{t}=c\left(z_{t}\right)$ are well-defined functions of $z_{t}$. Furthermore, they assume that $\operatorname{Pr}\left\{h_{t}^{k}>0\right\}=1$.

*This research was financially supported by the Jan Wallander's and Tom Hedelius' Foundation, Grant No. J03-41. The author thanks Pentti Saikkonen and Timo Teräsvirta for useful comments. Address correspondence to: Mika Meitz, Department of Economic Statistics, Stockholm School of Economics, P. O. Box 6501, SE-113 83 Stockholm, Sweden; e-mail: mika.meitz@hhs.se. 
Many GARCH(1,1) models are included in this family. For example, the choices $k=2, g_{t-1}=\alpha_{0}$, and $c_{t-1}=\beta+\alpha_{1} z_{t-1}^{2}$ yield the linear GARCH model of Bollerslev (1986). When $k=1, g_{t-1}=\alpha_{0}$, and $c_{t-1}=\beta+\alpha_{1}\left|z_{t-1}\right|$ the absolute value GARCH model of Taylor (1986) and Schwert (1989) is obtained. For $k=2, g_{t-1}=\alpha_{0}$, and $c_{t-1}=\beta+\left(\alpha_{1}+\omega I\left(z_{t-1}\right)\right) z_{t-1}^{2}$ (where $I\left(z_{t-1}\right)=1$ if $z_{t-1}<0$ and $I\left(z_{t-1}\right)=0$ otherwise) the model reduces to the GJR-GARCH model of Glosten, Jaganathan, and Runkle (1993). For a more extensive list, see He and Teräsvirta (1999a).

He and Teräsvirta (1999a) give conditions for the existence of moments of arbitrary order for the GARCH process (1)-(2). In particular, they show that the conditions $E\left[\left|z_{t}\right|^{k m}\right]<\infty$ and $E\left[c_{t}^{m}\right]<1$ are necessary and sufficient for the existence of the $k m$ th absolute moment of $\varepsilon_{t}, E\left[\left|\varepsilon_{t}\right|^{k m}\right]$. They also give an explicit formula for this moment under the stated conditions. He and Teräsvirta (1999b) consider a special case of the model (1)-(2), where it is assumed that $g_{t-1}=\alpha_{0}$ and $c_{t-1}$ has a particular parametric form. The exponent $k$ is no longer restricted to take one of the values 1 and 2 , but is only assumed to be a positive real number. They show that the conditions $E\left[\left|z_{t}\right|^{2 k}\right]<\infty$ and $E\left[c_{t}^{2}\right]<1$ are necessary and sufficient for the existence of $E\left[\left|\varepsilon_{t}\right|^{2 k}\right]$.

Ling and McAleer (2002) also considered the model (1)-(2) and complemented the results given in He and Teräsvirta (1999a, b). Ling and McAleer (2002) assume that $k$ is a positive real number and show that if $E\left[\left|z_{t}\right|^{k m}\right]<\infty, E\left[g_{t}^{m}\right]<\infty$, and $E\left[c_{t}^{m}\right]<1$ for some $m \in(0,1]$, then there exists a unique $k m$ th order stationary solution to (1)(2), which is also strictly stationary and ergodic. Furthermore, under the conditions $E\left[\left|z_{t}\right|^{k m}\right]<\infty$ and $E\left[g_{t}^{m}\right]<\infty$ and assuming now that $m$ is a positive integer, they show that the necessary and sufficient condition for the existence of the $k m$ th absolute moment of $\varepsilon_{t}, E\left[\left|\varepsilon_{t}\right|^{k m}\right]$, is $E\left[c_{t}^{m}\right]<1$ (in Theorem 2.2 of Ling and McAleer (2002) the exponent of $g_{t}$ and $c_{t}$ is $\mathrm{km}$ instead of $m$, but this appears to be a typographical error; the exponent appearing in their proof is $m$ ).

A yet unresolved issue is the necessary and sufficient condition for the existence of a strictly stationary solution to (1)-(2). In the case of a linear $\operatorname{GARCH}(1,1)$ model of Bollerslev (1986), this condition was derived in Nelson (1990). Bougerol and Picard (1992) extended this result to the linear $\operatorname{GARCH}(p, q)$ process. In both of these papers the condition is derived, in principle, using the theory of random matrices, and is formulated using the so called Lyapunov exponent.

In this short note we demonstrate that the same approach can be used to prove the necessity and sufficiency of a similar condition also in the case of the model (1)-(2). In fact, this readily follows from the results already given in Bougerol and Picard (1992) in the context of generalized autoregressive equations. 


\section{Main result}

We consider the case in which the exponent $k$ is assumed to be a positive real number (it plays no role in the following proof). First note that $\left\{g_{t}\right\}$ and $\left\{c_{t}\right\}$ are sequences of independent and identically distributed random variables because $\left\{z_{t}\right\}$ is. We assume that for all $t, g_{t}$ and $c_{t}$ are nonnegative and that either one of them is strictly positive with nonzero probability. This assumption is not very restrictive compared with the requirement $\operatorname{Pr}\left\{h_{t}^{k}>0\right\}=1$ made in He and Teräsvirta (1999a). From a practical point of view, most of the models listed as special cases in He and Teräsvirta (1999a) also satisfy this condition.

Rewriting (2) as

$$
h_{t}^{k}=g_{t-1}+c_{t-1} h_{t-1}^{k}, \quad t \in \mathbb{Z}
$$

makes it clear that the process $\left\{h_{t}^{k}\right\}$ follows an autoregressive equation in $\mathbb{R}^{+}$with independent and identically distributed nonnegative coefficients $g_{t}$ and $c_{t}$. Conditions for strict stationarity in such a situation are discussed in Bougerol and Picard (1992). The following result gives necessary and sufficient conditions for the existence of a strictly stationary solution of (3). We use the notation $\ln ^{+}(x)=\max \{\ln (x), 0\}$.

Theorem 1 (Corollary of Theorem 3.2 of Bougerol and Picard (1992)) Suppose that $E\left[\ln ^{+}\left(c_{t}\right)\right]$ is finite. If (3) has a strictly stationary nonnegative solution, then $E\left[\ln \left(c_{t}\right)\right]<$ 0 . Conversely, if $E\left[\ln ^{+}\left(g_{t}\right)\right]$ is finite and $E\left[\ln \left(c_{t}\right)\right]<0$, then for all $t \in \mathbb{Z}$, the series

$$
h_{t}^{k}=g_{t-1}+\sum_{k=1}^{\infty} c_{t-1} c_{t-2} \cdots c_{t-k} g_{t-k-1}
$$

converges a.s. and the process $\left\{h_{t}^{k}, t \in \mathbb{Z}\right\}$ is the unique strictly stationary solution of (3).

Proof. The assumption that either $g_{t}$ or $c_{t}$ is strictly positive with nonzero probability ensures that condition (C) of Bougerol and Picard (1992) is satisfied (see ibid., pp. 122123). In Theorem 3.2 of Bougerol and Picard (1992), the key condition for the existence of a strictly stationary solution is that the so called top Lyapunov exponent associated to a sequence of certain matrices is negative. In the present (univariate) case the top Lyapunov exponent equals

$$
\inf _{t \in \mathbb{N}} E\left[(t+1)^{-1} \ln \left(c_{0} c_{-1} \cdots c_{-t}\right)\right]=\inf _{t \in \mathbb{N}}\left\{(t+1)^{-1} \sum_{i=0}^{t} E\left[\ln \left(c_{-i}\right)\right]\right\}=E\left[\ln \left(c_{t}\right)\right] .
$$

The stated result now follows from Theorem 3.2 of Bougerol and Picard (1992).

The strict stationarity of the process $\left\{\varepsilon_{t}\right\}$ follows from that of $\left\{z_{t}\right\}$ and $\left\{h_{t}^{k}\right\}$. In the case of the linear $\operatorname{GARCH}(1,1)$ process, $g_{t}$ is a constant and the condition for $c_{t}$ has the 
form $E\left[\ln \left(\beta+\alpha z_{t}^{2}\right)\right]<0$, a condition already derived in Nelson (1990). The conditions for other members of the family of $\mathrm{GARCH}$ processes (1)-(2) are easily derived from Theorem 1.

Nelson (1990, Th. 6) derived an explicit expression for the moment $E\left[\ln \left(\beta+\alpha z_{t}^{2}\right)\right]$ in the case of standard normal or Cauchy errors using functions standard in the mathematical literature yet rather exotic in the econometric one. This derivation relies on the existence of explicit integral formulas for the logarithm of a polynomial (see the references in Nelson (1990)). Unfortunately, however, similar formulas for the moment $E\left[\ln \left(c_{t}\right)\right]$ do not seem to be available without making stringent assumptions about the functional form of $c(\cdot)$.

\section{References}

Bollerslev, T. (1986): "Generalized autoregressive conditional heteroskedasticity," Journal of Econometrics, 31, 307-327.

Bougerol, P., And N. Picard (1992): "Stationarity of GARCH processes and some nonnegative time series," Journal of Econometrics, 52, 115-127.

Glosten, L. W., R. Jaganathan, and D. E. Runkle (1993): "On the relation between the expected value and the volatility of the nominal excess return on stocks," Journal of Finance, 48, 1779-1801.

He, C., and T. Teräsvirta (1999a): "Properties of moments of a family of GARCH processes," Journal of Econometrics, 92, 173-192.

(1999b): "Statistical properties of the asymmetric power ARCH process," in Cointegration, Causality, and Forecasting: A Festschrift in Honour of Clive W. J. Granger, ed. by R. F. Engle, and H. White, pp. 462-474. Oxford University Press, Oxford.

Ling, S., And M. MCAleER (2002): "Stationarity and the existence of moments of a family of GARCH processes," Journal of Econometrics, 106, 109-117.

NeLson, D. (1990): "Stationarity and persistence in the $\operatorname{GARCH}(1,1)$ model," Econometric Theory, 6, 318-334.

SCHWERT, G. W. (1989): "Why does stock market volatility change over time?," Journal of Finance, 44, 1115-1153.

TAYlor, S. (1986): Modelling Financial Time Series. Wiley, New York. 